\title{
Rapid Reduction of Acute Subdural Hematoma and Redistribution of Hematoma -Case Report-
}

\author{
Arata WATAnABE*,**, Tomohiro OMATA**, and Hiroyuki KinOUCHI* \\ *Department of Neurosurgery, Faculty of Medicine, University of Yamanashi, Chuo, Yamanashi; \\ ${ }^{* *}$ Department of Neurosurgery, Tsuru Municipal Hospital, Tsuru, Yamanashi
}

\begin{abstract}
An 88-year-old woman presented with acute subdural hematoma (ASDH) which showed rapid resolution on computed tomography (CT) and magnetic resonance (MR) imaging. She was transferred to our hospital after falling out of bed. On admission, she was comatose with Japan Coma Scale score of 200 and Glasgow Coma Scale score of E1V1M2. Brain CT showed a thick left frontotemporal ASDH. Conservative treatment consisted of $200 \mathrm{ml}$ of glycerol administered intravenously twice a day, and maintenance in the approximately 20 degree head-up position to reduce intracranial pressure. Three days later, her consciousness recovered to Japan Coma Scale score of 30 and Glasgow Coma Scale score of E2V4M5. CT showed obvious reduction of the hematoma without brain or scalp swelling. Spinal MR imaging detected no redistribution of hematoma to the spine. The present case illustrates that rapid spontaneous reduction of ASDH may occur by redistribution of hematoma, mainly to the supratentorial subdural space because of brain atrophy.
\end{abstract}

Key words: acute subdural hematoma, brain atrophy, mechanism, rapid spontaneous reduction, redistribution

\section{Introduction}

Rapid resolution of acute subdural hematoma (ASDH) on computed tomography (CT) and magnetic resonance (MR) imaging is a known phenomenon, ${ }^{17)}$ with a number of reported cases. ${ }^{1,5,13,14,16,19)}$ Four mechanisms for this rapid resolution of hematoma have been proposed: Dilution and wash out of the hematoma by cerebrospinal fluid (CSF) af-

Received December 3, 2009;

Accepted February 10, 2010 ter tearing of the arachnoid membrane ${ }^{13,15,18)}$; compression and redistribution of the hematoma by acute brain swelling $8,11,13,17)$; redistribution of the hematoma through skull fractures ${ }^{2,10,12,21)}$; and redistribution of the hematoma to the spinal subdural space..$^{3,20)}$

Here we report a case of rapid spontaneous reduction of ASDH due to redistribution of hematoma, mainly to the supratentorial subdural space because of the brain atrophy. 

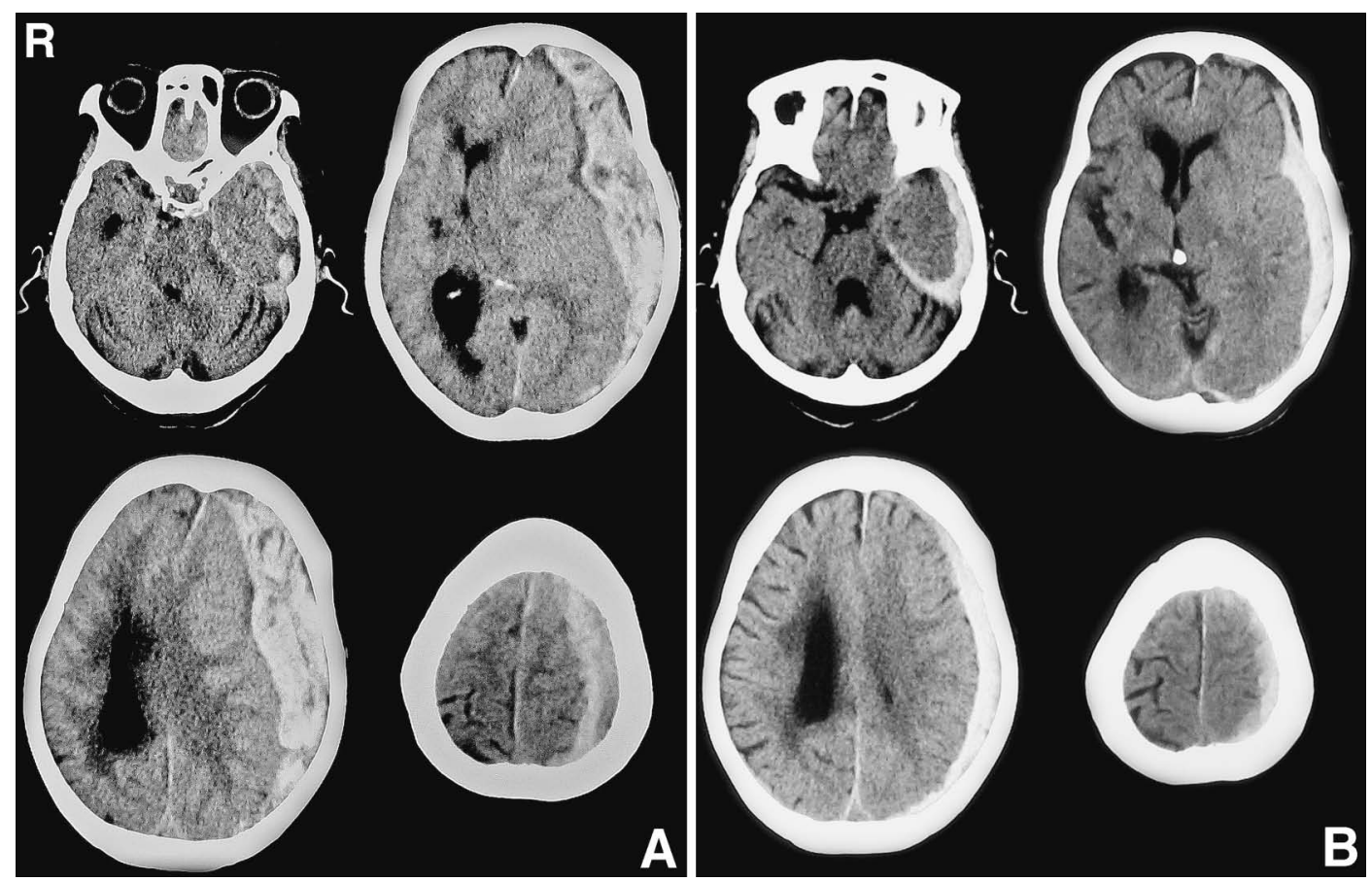

Fig. 1 A: Initial computed tomography (CT) scans demonstrating a thick acute subdural hematoma in the left frontotemporal lobe with herniation sign, compression and tilting of the brain stem, and dilation of the right lateral ventricle. B: Second CT scans 3 days after admission showing obvious reduction of the acute subdural hematoma, and disappearance of the compression of the brain stem.

\section{Case Report}

An 88-year-old woman was transferred to our hospital after falling out of bed. On admission, she was comatose with Japan Coma Scale score of 200 and Glasgow Coma Scale score of E1V1M2. The left parietal skin was slightly reddish without swelling. Initial brain CT showed a thick left frontotemporal ASDH with herniation sign (Fig. 1A). Bone window CT and skull radiography did not show any skull fracture. She had a history of cerebral infarction resulting in severe left hemiparesis, chronic heart failure, and dementia.

Considering her advanced age, poor general condition, and family wishes, we did not perform surgery to evacuate the hematoma under general anesthesia. She was treated with $200 \mathrm{ml}$ of glycerol administered intravenously twice a day, and kept in the approximately 20 degree head-up position to reduce intracranial pressure. Three days later, her consciousness recovered to Japan Coma Scale score of 30 and Glasgow Coma Scale score of E2V4M5. CT showed obvious spontaneous reduction of the hematoma without brain or scalp swelling (Fig. 1B). Axial and coronal reconstruction of conventional CT scans showed redistribution of hematoma to the supratentorial subdural space (Fig. 2A-C). Brain sagittal MR imaging also showed redistribution of hematoma to the supratentorial subdural space (Fig. 2D). Whole spinal MR imaging detected no redistribution of the hematoma to the spinal subdural space (Fig. 2E). Finally, the ASDH transformed to chronic subdural hematoma, and simple drainage was performed 3 weeks after the initial head injury. The patient is now confined to a wheelchair.

\section{Discussion}

The present case of rapid spontaneous reduction of ASDH was characterized by redistribution of the hematoma, mainly to the supratentorial subdural space. We suggest that dissociation between the skull and brain may have been important in this movement of hematoma,4) by providing the space needed for the redistribution. The majority of reported cases including our case of rapid spontaneous resolution of ASDH occurred in elderly patients ${ }^{6}$ or infants. ${ }^{7)}$ Brain swelling may be important in the redistribution of hematoma in young patients. ${ }^{9}$

In the present case, compression and redistribution of the relatively thin hematoma by acute brain swelling, ${ }^{8,11,13,17)}$ redistribution of the hematoma through a skull fracture, $, 2,10,12,21)$ redistribution of the hematoma to the spinal subdural space, ${ }^{3,20)}$ and dilution and washing out of the hematoma by CSF after tearing of the arachnoid membrane ${ }^{13,15,18)}$ are all possible mechanisms for the spontaneous resolution of the ASDH, but were not considered to be the main mechanism for rapid reduction of ASDH.

CT demonstrated a low density band between the hematoma and the inner wall of the skull bone, which indicated co-mingling of the hematoma with CSF. ${ }^{18)}$ The majority of cases of spontaneous ASDH resolution were associated with such a low density band, ${ }^{11)}$ including our case. The 

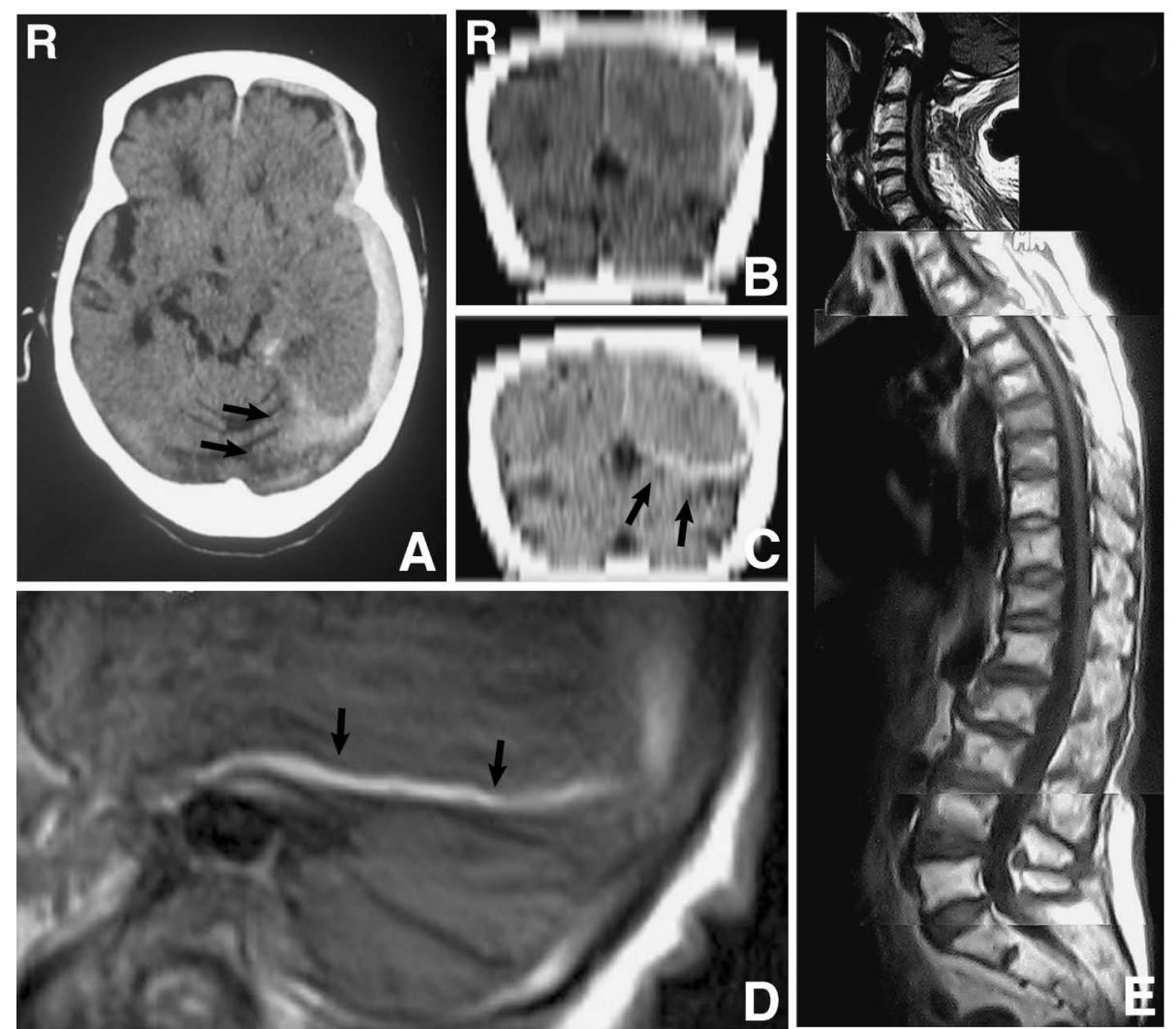

Fig. 2 A: Axial computed tomography (CT) scan showing redistribution of hematoma to the supratentorial subdural space (arrows). B: Reconstructed conventional CT scan on admission demonstrating thick acute subdural hematoma. C: Second CT scan showing hematoma redistribution to the supratentorial subdural space, which was thick enough to explain the significant reduction of the convexity subdural hematoma (arrows). D: Brain sagittal $T_{1}$-weighted magnetic resonance (MR) image showing redistribution of hematoma to the supratentorial subdural space (arrows). E: Whole spine $T_{1}$-weighted MR images showing no redistribution of the hematoma to the spinal subdural space.

most important factors in rapid ASDH resolution appear to be participation of the CSF and presence of a wide subdural space in which the hematoma can be redistributed. ${ }^{11)}$ We also suggest that these two factors are the most important in the rapid resolution of ASDH.

Emergent evacuation of hematoma is the gold standard for patients with ASDH, but we should not exclude treatment for patients who cannot undergo surgery for various reasons. Some patients may experience spontaneous resolution of the hematoma resulting in good outcome. Patients with brain atrophy and the indicative low density band on CT may undergo spontaneous resolution of ASDH, but emergent surgery should not be delayed if possible.

The present case of rapid spontaneous reduction of ASDH was due to redistribution of the hematoma, mainly to around the tentorium because of brain atrophy. Rapid resolution of ADSH may occur by redistribution of hematoma to the supratentorial subdural space as a result of brain atrophy, as well as dilution and washing out by CSF.

\section{Acknowledgment}

We thank Mrs. Ichikawa, Saigusa, Nakamura, and
Mizukoshi for their assistance in collecting radiographical data.

\section{References}

1) Aoki N: Acute subdural haematoma with rapid resolution. Acta Neurochir (Wien) 103: 76-78, 1990

2) Berker M, Gulsen S, Ozcan OE: Ultra rapid spontaneous resolution of acute posttraumatic subdural hematomas in patient with temporal linear fracture. Acta Neurochir (Wien) 145: 715-717, 2003

3) Bortolotti C, Wang H, Fraser K, Lanzino G: Subacute spinal subdural hematoma after spontaneous resolution of cranial subdural hematoma: causal relationship or coincidence? Case report. J Neurosurg 100: 372-374, 2004

4) Cohen JE, Eger K, Montero A, Israel Z: Rapid spontaneous resolution of acute subdural hematoma and HIV related cerebral atrophy: case report. Surg Neurol 50: 241-244, 1998

5) Cuatico W, Yamamoto R, Howeiler B, Smith R: Spontaneous resolution of subdural hematomas. J Neurosurg Sci 35: 139-145, 1991

6) Fujioka S, Hamada J, Kaku M, Ushio Y: [Rapid resolution of acute subdural hematoma. Report of two cases]. Neurol Med Chir (Tokyo) 30(11 Spec No): 827-831, 1990 (Japanese)

7) Huang SH, Lee HM, Lin CK, Kwan AL, Howng SL, Loh JK: 
Rapid resolution of infantile acute subdural hematoma: a case report. Kaohsiung J Med Sci 21: 291-294, 2005

8) Inamasu J, Nakamura Y, Saito R, Kuroshima Y, Mayanagi K, Ohba S, Ichikizaki K: Rapid resolution of traumatic acute subdural hematoma by redistribution. Am J Emerg Med 20: 376-377, 2002

9) Joki $T$, Hashimoto $T$, Akachi $K$, Boku M, Suzuki $K$, Nakamura N: [Rapid resolution of acute subdural hematoma; report of two cases]. No Shinkei Geka 20: 915-919, 1992 (Japanese)

10) Kang SH, Chung YG, Lee HK: Rapid disappearance of acute posterior fossa epidural hematoma. Neurol Med Chir (Tokyo) 45: 462-463, 2005

11) Kato N, Tsunoda T, Matsumura A, Yanaka K, Nose T: Rapid spontaneous resolution of acute subdural hematoma occurs by redistribution-two case reports. Neurol Med Chir (Tokyo) 41: 140-143, 2001

12) Kundra SN, Kundra R: Extracranial redistribution causing rapid spontaneous resolution of acute subdural hematoma. Neurol India 53: 124, 2005

13) Matsuyama T, Shimomura T, Okumura $Y$, Sakaki T: Rapid resolution of symptomatic acute subdural hematoma: case report. Surg Neurol 48: 193-196, 1997

14) Mirzai H, Yaldiz C, Eminoglu M, Orguc S: Neurological Picture. Ultra fast resolution of acute post-traumatic subdural haematoma. J Neurol Neurosurg Psychiatry 76: 1738, 2005

15) Nagao T, Aoki N, Mizutani H, Kitamura K: Acute subdural hematoma with rapid resolution in infancy: case report. Neurosurgery 19: 465-467, 1986
16) Niikawa S, Sugimoto S, Hattori T, Ohkuma A, Kimura T, Shinoda J, Funakoshi T: Rapid resolution of acute subdural hematoma-report of four cases. Neurol Med Chir (Tokyo) 29: 820-824, 1989

17) Polman CH, Gijsbers CJ, Heimans JJ, Ponssen H, Valk J: Rapid spontaneous resolution of an acute subdural hematoma. Neurosurgery 19: 446-448, 1986

18) Suzuki Y, Kawamata T, Matsumoto H, Kunii N, Matsumoto $\mathrm{K}$ : [A resolving sign of acute subdural hematoma: from report of two cases]. No Shinkei Geka 26: 1025-1029, 1998 (Japanese)

19) Tsui EY, Fai Ma K, Cheung YK, Chan JH, Yuen MK: Rapid spontaneous resolution and redistribution of acute subdural hematoma in a patient with chronic alcoholism: a case report. Eur J Radiol 36: 53-57, 2000

20) Wong ST, Yuen MK, Fok KF, Yuen SC, Yam KY, Fong D: Redistribution of hematoma to spinal subdural space as a mechanism for the rapid spontaneous resolution of posttraumatic intracranial acute subdural hematoma: case report. Surg Neurol 71: 99-102, 2009

21) Wu MC, Liu JX, Luo GC, Zhang ZW, Min J, Yu H, Yao Y: Rapid natural resolution of intracranial hematoma. Chin J Traumatol 7: 96-100, 2004

Address reprint requests to: Arata Watanabe, M.D., Ph.D., Department of Neurosurgery, Tsuru Municipal Hospital, 5-1-55 Tsuru, Tsuru, Yamanashi 402-0056, Japan.

e-mail: arata@yamanashi.ac.jp 